\title{
Using population-based data to predict the impact of introducing noninvasive prenatal diagnosis for Down syndrome
}

\author{
Marleen R. Susman, MSc, ${ }^{1}$ David J. Amor, FRACP, PhD, ${ }^{2}$ Evelyne Muggli, MPH, ${ }^{1}$ \\ Alice M. Jaques, $P h D,^{1}$ and Jane Halliday, $P h D^{1}$
}

\begin{abstract}
Purpose: To compare the number and types of chromosome abnormalities prenatally diagnosed and the number of invasive procedures between current prenatal testing pathways and a pathway where noninvasive prenatal diagnosis for Down syndrome replaces Down syndrome screening tests. Methods: Numbers and types of chromosome abnormalities for each referral category were extracted from prenatal diagnostic testing reports routinely collected in Victoria, Australia, in 2006 and 2007. These data were then applied to the proposed implementation strategy. Results: If noninvasive prenatal diagnosis for Down syndrome had replaced Down syndrome screening tests in 2006 and 2007, in Victoria, there would have been $25(7 \%)$ additional Down syndrome diagnosed, 6896 (84\%) fewer invasive procedures, and 231 (56\%) non-Down syndrome chromosome abnormalities no longer detected. These include trisomy 13, trisomy 18, sex chromosome abnormalities, balanced and unbalanced rearrangements, polyploidy, and mosaic results. Conclusions: The potential loss of information about chromosome abnormalities other than Down syndrome with noninvasive prenatal diagnosis compared with full karyotyping with traditional prenatal diagnosis should be considered when planning for the implementation of new technologies. Genet Med 2010:12(5):298-303.
\end{abstract}

Key Words: chromosome aberrations, Down syndrome, karyotyping, noninvasive, prenatal diagnosis

$\mathrm{N}$ oninvasive prenatal diagnosis (NIPD) for chromosome abnormalities has the potential to revolutionize prenatal diagnosis in the next 5 years. The discovery of cell-free fetal nucleic acids in maternal serum ${ }^{1,2}$ has accelerated the development of NIPD as a diagnostic test, and NIPD strategies are currently being investigated only for trisomy 21 (Down syndrome $)^{3-9}$ or for trisomies 13,18 , and $21 . .^{10,11}$ Ways of introducing routine screening of chromosome abnormalities using NIPD and the implications of different strategies have not been discussed.

In Victoria, Australia, as in many other places around the world, women currently self-select to have invasive diagnostic testing (chorionic villus sampling or amniocentesis) and karyotyping based on real or perceived risks of Down syndrome and other chromosome abnormalities. Most of these women have an

From ${ }^{1}$ Public Health Genetics, and ${ }^{2}$ Genetic Health Services Victoria, Murdoch Childrens Research Institute, Royal Children's Hospital, Parkville, Victoria, Australia

Marleen Susman, MSc, Public Health Genetics, Murdoch Childrens Research Institute, Royal Children's Hospital, Flemington Road, Parkville, Victoria 3052, Australia. E-mail: marleen.susman@mcri.edu.au.

Disclosure: The authors declare no conflict of interest..

Submitted for publication December 1, 2009.

Accepted for publication January 22, 2010.

Published online ahead of print March 5, 2010.

DOI: $10.1097 /$ GIM.0b013e3181d5d022 increased risk of Down syndrome based on their age, results of first trimester combined serum screening (1TCS), second trimester maternal serum screening (2TS), or increased nuchal translucency alone. Karyotyping is a genome-wide test, evaluating all 23 pairs of chromosomes for their number, size, shape, and banding pattern, to detect structural and numerical chromosome abnormalities. Importantly, karyotyping detects a far broader spectrum of chromosome abnormalities than Down syndrome.

NIPD for Down syndrome promises a decrease in the number of invasive tests and an increase in the prenatal detection of Down syndrome. However, there is also the potential for a decrease in the detection of chromosome abnormalities other than Down syndrome. ${ }^{12}$ In Victoria, with $\sim 70,000$ births each year, we can examine the existing situation using populationbased data that are routinely collected for all prenatal screening and prenatal diagnostic tests. To predict the potential effect of NIPD for Down syndrome on prenatal testing outcomes, the data can then be applied to one potential implementation model in which NIPD for Down syndrome replaces serum screening tests.

The main aims of this study were to predict changes in the detection of chromosome abnormalities and in the number of invasive procedures if NIPD for Down syndrome was introduced in our setting. This analysis will provide a basis for gauging the potential effect of implementing NIPD for Down syndrome and inform consideration of the advantages of current practices balanced against the advantages of potential new strategies.

\section{METHODS}

Two population-based data sets were used:

1. Genetic health prenatal screening database: records for 2006 and 2007 of all women in Victoria who had either first trimester combined screening (1TCS) or second trimester screening (2TS).

2. Victorian prenatal diagnosis database: records for 2006 and 2007 from all four cytogenetic laboratories in Victoria that process prenatal diagnostic tests, including karyotype results and indications for testing. ${ }^{13,14}$

\section{Indications}

For all prenatal cytogenetic tests, the indications for testing were grouped into the following categories: age (maternal age, $\geq 37$ years), screening tests (1TCS, 2TS, and nuchal translucency screening), abnormal ultrasound (fetal anomaly scan between 16 and 20 weeks), carrier parent (known carrier of a balanced chromosomal rearrangement), and other (maternal age $<37$ years, couples who had a previous chromosomally abnormal aneuploid pregnancy, and psychosocial reasons).

\section{Karyotype results}

Karyotype results were grouped into the following categories: no abnormality detected, Down syndrome, other autosomal tri- 


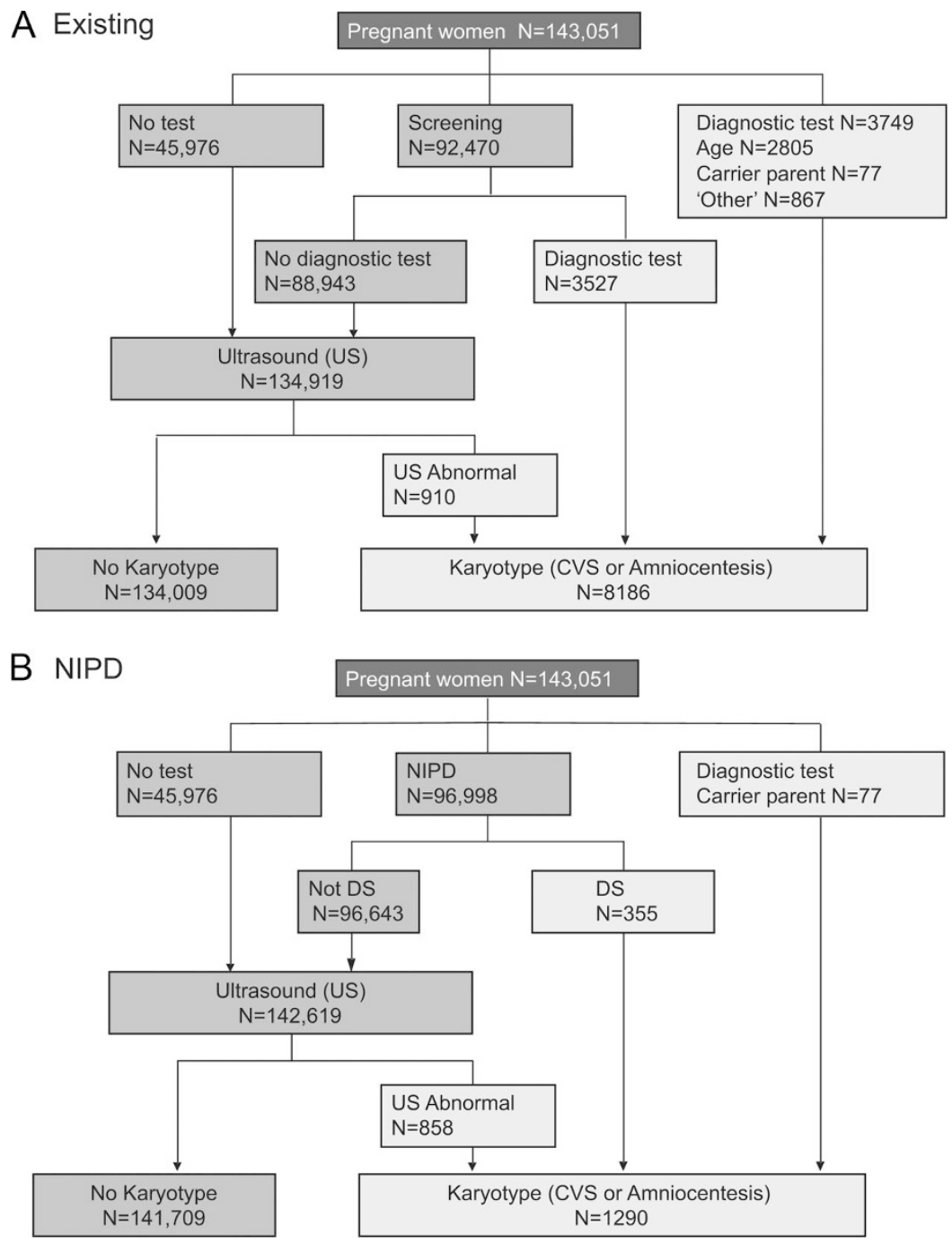

Fig. 1. Testing pathways: A, Existing and B, NIPD.

somies, sex chromosome aneuploidy, polyploidy, unbalanced rearrangements, balanced rearrangements, and mosaicism. Only level III mosaic results, representing true mosaicism, were included.

\section{NIPD}

Although recognizing that there are technical aspects of NIPD that require refinement, ${ }^{15,16}$ we assumed an extremely high sensitivity and specificity, and applicability to all women. We also assumed that although free fetal DNA is present in maternal serum as early as 7 weeks gestation, ${ }^{17}$ the test would be offered at a more realistic 12 weeks gestation to coincide with the gestation at which 1TCS and chorionic villous sampling (CVS) are offered.

\section{Prenatal pathways}

Figure 1 shows the existing testing pathways and the proposed NIPD pathways in which screening tests for Down syndrome are replaced with NIPD for Down syndrome. The NIPD pathways were constructed so that: a. women with a referral indication of carrier parent go directly (without previous NIPD) to an invasive test and karyotyping;

b. women who have no testing with existing pathways continue to have no testing;

c. women with referral indications of screening tests, age, and other, with existing pathways, have NIPD for Down syndrome (receiving either a Down syndrome or not Down syndrome result);

d. women continue to have fetal anomaly scans at 18 weeks (same as existing pathways);

e. women with abnormal ultrasound findings may have invasive testing and karyotyping (same as existing pathways); and

f. women with Down syndrome diagnoses after NIPD have invasive testing and karyotyping for confirmation.

\section{Calculations}

The increase in the number of Down syndrome diagnoses with NIPD should be equivalent to the number of false-negative results with $1 \mathrm{TCS}$ and $2 \mathrm{TS}$. To calculate the number of false 


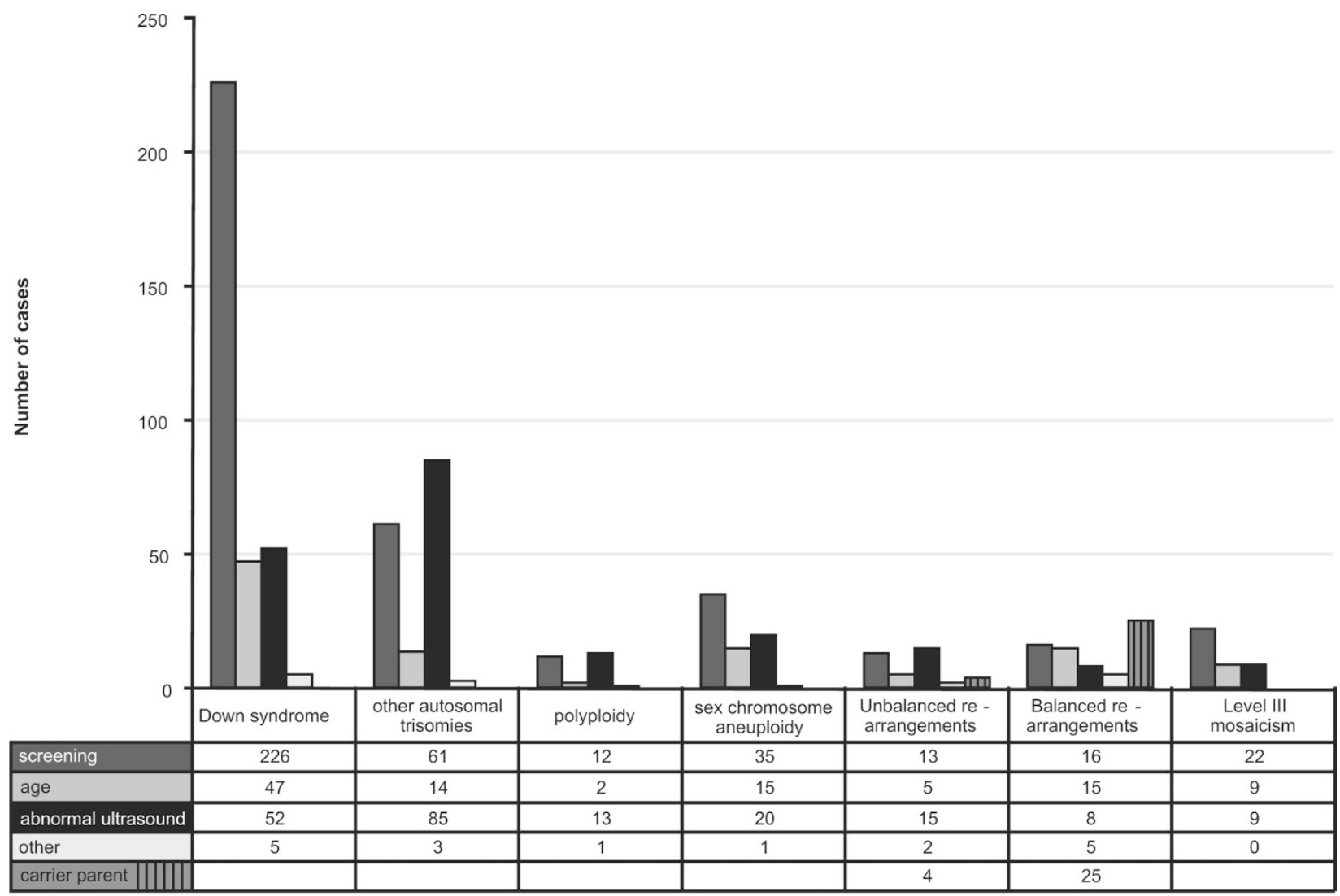

Abnormal chromosome result category

Fig. 2. Chromosome abnormalities detected from each referral category with existing pathways.

negatives $\left(\mathrm{DS}_{\mathrm{n}}\right)$, we combined the number of Down syndrome diagnoses from referral indications of 1TCS and 2TS with published sensitivities for our population of $90.5 \%$ for $1 \mathrm{TCS}^{18}$ and $85 \%$ for 2 TS. ${ }^{19}$ The false-negative results refer to women who had 1TCS or 2TS, no diagnostic test, but had a pregnancy with Down syndrome. It includes fetal deaths, terminations, and livebirths. With NIPD for Down syndrome, these women would have received a diagnosis instead of a risk figure. If $\mathrm{DS}_{t}$ is the total number of Down syndrome detected by the NIPD pathways, and $\mathrm{DS}_{\mathrm{e}}$ is the number of Down syndrome detected with existing pathways, then $\mathrm{DS}_{\mathrm{t}}=\mathrm{DS}_{\mathrm{n}}+\mathrm{DS}_{\mathrm{e}}$.

The number of non-Down syndrome abnormalities detected with the NIPD pathways were calculated by combining the number of non-Down syndrome abnormal results in the carrier parent and abnormal ultrasound referral groups (Fig. 1, B). The number of invasive tests with the NIPD pathways equals the number of referrals for carrier parent, Down syndrome diagnoses after NIPD, and abnormal ultrasound (Fig. 1, B).

\section{RESULTS}

\section{Existing pathways}

The total number of referrals for karyotyping in 2006 and 2007 was 8186 . Data were combined for CVS and amniocentesis, with $33 \%$ of procedures being CVS. The total number of abnormal results was 740 (9\%), comprising 330 Down syndrome results and 410 abnormal non-Down syndrome results. Referrals for prenatal diagnosis were screening 3527 (43\%), age
2805 (34\%), abnormal ultrasound 910 (11\%), other 867 (10\%), and carrier parent $77(1 \%)$.

Figure 2 shows the breakdown by category of chromosome abnormality for each referral indication with existing pathways. For all indications, a high proportion of abnormal results were non-Down syndrome. When the indication was screening, 159 of $385(41 \%)$ of the abnormal results were non-Down syndrome, whereas for all other indication categories, non-Down syndrome abnormalities were more frequent than Down syndrome: carrier parent 29 of $29(100 \%)$, abnormal ultrasound 150 of $202(74 \%)$, age 60 of $107(61 \%)$, and 'other' 12 of $17(70 \%)$.

\section{NIPD pathways}

The additional number of Down syndrome detected with the NIPD pathways was calculated as 25 , comprising 21 undiagnosed Down syndrome pregnancies after 1TCS and 4 after 2TS. Therefore, the Down syndrome diagnoses would have increased from the existing 330 to 355 , a $7 \%$ increase (Fig. 3).

Figure 3 also compares the number of non-Down syndrome abnormalities detected between existing and NIPD pathways. With existing pathways, there were 410 non-Down syndrome abnormalities, whereas with NIPD, there would have been 179 , a reduction of $56 \%$. The 231 cases no longer detected with NIPD comprised 78 (34\%) other autosomal aneuploidies (76 of 78 were either trisomy 18 or trisomy 13$), 51$ (22\%) sex chromosome abnormalities, $36(16 \%)$ balanced rearrangements, 31 (13\%) mosaic results, 20 (9\%) unbalanced rearrangements, and $15(6 \%)$ polyploidy. 


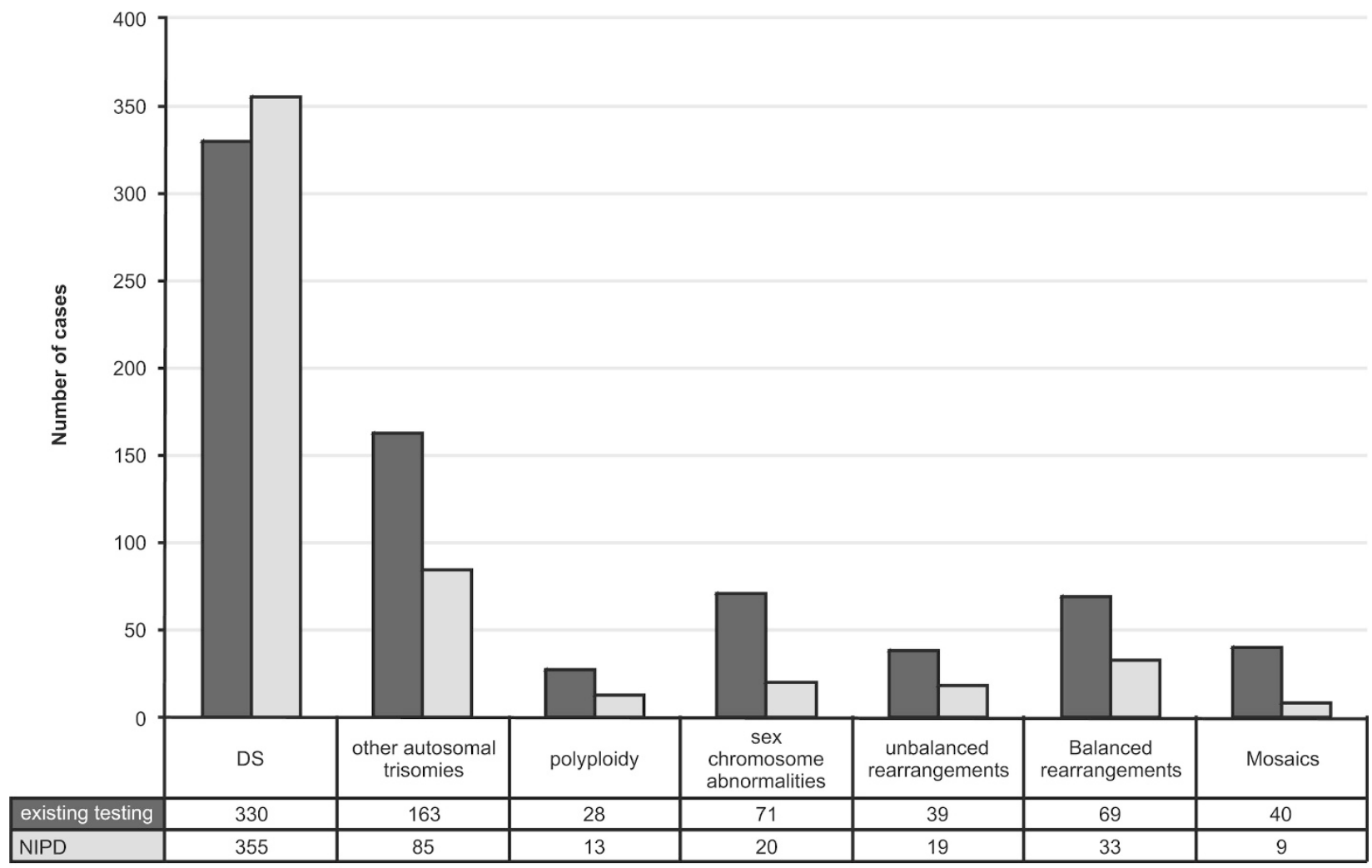

Abnormal chromosome result category

Fig. 3. Comparing chromosome abnormalities detected between existing testing pathways and NIPD.

Overall, the replacement of screening tests with NIPD for Down syndrome would have resulted in a $28 \%$ reduction in the detection of all chromosome abnormalities, a decrease from the existing 740 to 534 abnormalities.

If NIPD was extended to include testing for chromosomes 13 and 18 (in addition to Down syndrome), the number of other abnormalities no longer detected would reduce from 231 to 155 . This would be an overall reduction in chromosome abnormalities of $17 \%$, from 740 to 610 (includes 25 extra cases of Down syndrome). If sex chromosome abnormalities were also included, the reduction in the number of abnormalities no longer detected would reduce from 231 to 104 , and an overall reduction of $11 \%$, from 740 to 661 .

\section{Invasive testing}

Figure 1 also shows that with the NIPD pathways, the number of invasive tests would have reduced from the existing 8186 to 1290 , a reduction of $84 \%$. The 1290 invasive procedures comprise 77 carrier parent referrals, 355 Down syndrome confirmations after NIPD, and 858 abnormal ultrasound referrals. Based on current detection rates, the 858 abnormal ultrasound referrals represent the minimum number of referrals for CVS and amniocentesis. The proportion of abnormal karyotype results with existing testing is 740 of $8186(9 \%)$, rising to 534 of $1290(41 \%)$ with these NIPD pathways.

If testing for trisomy 13 and trisomy 18 were included in a NIPD panel, and confirmatory invasive testing of abnormal results were required, there would have been an additional 76 invasive procedures, taking the total to 1366 . If sex chromosome aneuploidy was also included with abnormal confirmatory invasive testing, there would have been a total of 1417 invasive procedures, a reduction of $83 \%$.

\section{DISCUSSION}

The major strength of this study is that we have based our analysis on actual population-based data to explore the different types of chromosome abnormalities that will no longer be detected by NIPD. Our results show that implementation of NIPD for Down syndrome, as a replacement for Down syndrome serum screening tests, will have a significant impact on the prenatal detection of chromosome abnormalities and the number of invasive procedures. Although there are still uncertainties about how NIPD will be implemented, our analysis provides a basis from which different implementation strategies can be considered to ensure that the advantages of current practices are balanced against the advantages of potential new strategies. We have shown that in our population, if NIPD had been introduced in 2006 and 2007, the detection of Down syndrome would have increased by a relatively modest $7 \%$, with a much greater benefit being the $84 \%$ reduction in invasive procedures. We have also shown that NIPD for Down syndrome would have resulted in a $28 \%$ reduction in the detection of all chromosome abnormalities. Specifically, there would have been a $56 \%$ reduction in the detection of non-Down syndrome chromosome abnormalities.

These results highlight the main benefit of NIPD in terms of the anticipated reduction in the number of invasive procedures. This will be welcomed by many pregnant women, who currently experience anxiety and stress associated with weighing 
their risk of a Down syndrome pregnancy against the risks of miscarriage with invasive testing. ${ }^{20,21}$ However, this benefit must also be considered in the context of the predicted small increase in the detection of Down syndrome and the large reduction in the detection of non-Down syndrome chromosome abnormalities.

The clinical outcome of these abnormalities varies: the $40 \%$ that are autosomal trisomies or polyploidy typically result in miscarriage, stillbirth, or neonatal death; sex chromosome abnormalities $(22 \%)$ will vary in severity, with some compatible with normal health and lifespan and others leading to developmental and reproductive problems ${ }^{22}$; unbalanced chromosome rearrangements and mosaics $(22 \%)$ are more likely to result in the birth and long-term survival of a child with a disability ${ }^{23}$; and apparently balanced chromosome rearrangements $(16 \%)$ are unlikely to lead to health problems in the child but may have implications for the future reproductive risks for the parents and the child. ${ }^{24}$ The importance for women of these clinical outcomes is unknown.

Some pregnancies with chromosome abnormalities that are missed by NIPD will have fetal structural abnormalities detectable on ultrasound. It is expected that in a proportion of these pregnancies, the detection of fetal abnormality will trigger amniocentesis, resulting in diagnosis of the chromosome abnormality. However, determining the number of abnormalities that will be diagnosed by this pathway is not possible with current data. In particular, we do not know the proportion of pregnancies that will have detectable structural abnormalities. A recent review of second trimester ultrasound markers for fetal aneuploidy suggests that many will not have significant findings, ${ }^{25}$ and even in high-risk populations only one third of chromosome abnormalities may be detectable by ultrasound. ${ }^{26}$ The competency of the ultrasonologist and the equipment used will also affect the results. The other unknown factor is the proportion of women who will elect to have amniocentesis following the ultrasound detection of a structural abnormality, particularly in the setting of a previous normal NIPD test.

The importance to pregnant women of diagnosing non-Down syndrome chromosome abnormalities has been explored in the context of offering targeted molecular testing for chromosomes $13,18,21, \mathrm{X}$ and $\mathrm{Y}$ (aneuploidy screening), instead of karyotyping on invasive specimens. ${ }^{27-30}$ The results are inconsistent, indicating that attitudes of pregnant women may be highly variable. Of particular relevance to NIPD for Down syndrome, one study showed that neither health professionals nor women were in favor of a test for Down syndrome alone. ${ }^{27}$

With the inclusion of trisomy 13 , trisomy 18 , and sex chromosome aneuploidy in a NIPD panel, the number of missed abnormalities would decrease from $28-11 \%$ of current abnormalities detected. This would not be at the expense of the large reduction in invasive procedures that would only change slightly from an $84 \%$ reduction with NIPD for Down syndrome to $83 \%$ with an aneuploidy panel. This is a benefit worth considering.

Women have shown an overall positive attitude toward the introduction of NIPD, but this may be in the absence of an informed understanding of the consequences and choices they may be confronted with. ${ }^{31}$ Women preferring to receive results for targeted NIPD testing may see the removal of the perceived uncertainty and difficulty in counseling and decision making for unexpected abnormalities as a benefit. ${ }^{32}$ Other women may have a need to maximize their information seeking and will choose invasive testing. Undoubtedly, all stakeholders should participate in deciding what tests are available and how both management and counseling of pregnant women should be addressed. ${ }^{12,32,33}$

A limitation of this study is the need to make certain assumptions about NIPD to predict its impact. These assumptions relate to the robustness and accuracy of the test, and the choices that women will make if NIPD replaces screening tests, especially with regard to women currently having no testing and continuing to have no testing. There are four groups of women currently not having invasive prenatal testing who may choose NIPD: those that do not want invasive testing, those that present too late in pregnancy, those that miscarry before testing but had intended to be tested, and those not offered testing. Some of these pregnancies will be chromosomally abnormal, including Down syndrome pregnancies, which would increase the number of additional Down syndrome detected with NIPD. However, it would not affect the reduction in the proportion of non-Down syndrome abnormalities detected.

Another limitation is that our results reflect existing prenatal practices in Victoria, where $33 \%$ of invasive procedures are CVS that detects a higher rate of chromosome abnormalities. Other settings with different prenatal practices will also experience a reduction in the number of non-Down syndrome abnormalities detected, although the actual numbers may differ from ours.

In conclusion, our results predict that the implementation of NIPD for Down syndrome will lead to a major reduction in the number of invasive procedures but will only detect a small number of additional Down syndrome pregnancies compared with the current Down syndrome screening and diagnostic testing programs. NIPD will also lead to a reduction in the detection of non-Down syndrome chromosome abnormalities. The number not detected would be reduced if other aneuploidies are included in an NIPD panel. Our data, showing the effects of two possible implementation strategies for NIPD for chromosome abnormalities, provide a platform for continuing informed discussion to take place to address the relevance and significance of these non-Down syndrome abnormalities for all stakeholders, especially pregnant women, before any implementation of NIPD.

\section{ACKNOWLEDGMENTS}

Marleen Susman is the recipient of an Australian Postgraduate Award Scholarship from the University of Melbourne. Jane Halliday has a National Health and Medical Research Council Senior Research Fellowship (436904). The authors thank Leonard Bonacquisto for supplying the data from the Genetic Health prenatal screening database.

The primary purpose of the prenatal diagnosis database is to monitor and evaluate trends and outcomes in this sector of health care. This activity is considered to be quality assurance. From time to time research projects arising from the prenatal database are approved by Human Research Ethics Committees. However, in accordance with specific advice issued by Australia's National Health and Medical Research Council, this study was not deemed research, because the activity was a noninterventional evaluation/model, there was no potential for breach of confidentiality or privacy as the data were supplied to the primary investigator in a de-identified manner and it presented no other ethical issues.

\section{ACKNOWLEDGMENTS}

Marleen Susman wrote the article, designed the study, analyzed the deidentified prenatal diagnostic data, and provided the interpretation and discussion of results. Marleen Susman had full access to all of the data used in the study and takes 
responsibility for the integrity of the data and the accuracy of the data analysis. David Amor was the clinical expert who discussed and advised on all aspects of the article, especially the discussion of results. Evelyne Muggli was involved in the collection of the prenatal diagnostic data and provided advice on analysis of the data. Alice Jaques provided critical discussions relating to the interpretation and discussion of results. Jane Halliday provided expertise in epidemiology, as well as discussion and advice on all aspects of the article.

\section{REFERENCES}

1. Lo YM, Corbetta N, Chamberlain PF, et al. Presence of fetal DNA in maternal plasma and serum. Lancet 1997;350:485-487.

2. Poon LL, Leung TN, Lau TK, Lo YM. Presence of fetal RNA in maternal plasma. Clin Chem 2000;46:1832-1834.

3. Benachi A, Costa JM. Non-invasive prenatal diagnosis of fetal aneuploidies. Lancet 2007;369:440-442.

4. Chim SS, Jin S, Lee TY, et al. Systematic search for placental DNAmethylation markers on chromosome 21: toward a maternal plasma-based epigenetic test for fetal trisomy 21. Clin Chem 2008;54:500-511.

5. Dhallan R, Guo X, Emche S, et al. A non-invasive test for prenatal diagnosis based on fetal DNA present in maternal blood: a preliminary study. Lancet 2007;369:474-481.

6. Hulten MA, Old RW. Non-invasive prenatal diagnosis of Down's syndrome. Lancet 2007;369:1997; author reply 1998-1999.

7. Lo YM, Lun FM, Chan KC, et al. Digital PCR for the molecular detection of fetal chromosomal aneuploidy. Proc Natl Acad Sci U S A 2007;104: 13116-13121.

8. Lo YM, Tsui NB, Chiu RW, et al. Plasma placental RNA allelic ratio permits noninvasive prenatal chromosomal aneuploidy detection. Nat Med 2007; $13: 218-223$.

9. Lo YMD. Noninvasive prenatal detection of fetal chromosomal aneuploidies by maternal plasma nucleic acid analysis: a review of the current state of the art. BJOG 2009;116:152-157.

10. Chiu RW, Chan KC, Gao Y, et al. Noninvasive prenatal diagnosis of fetal chromosomal aneuploidy by massively parallel genomic sequencing of DNA in maternal plasma. Proc Natl Acad Sci U S A 2008;105:20458-20463.

11. Fan HC, Blumenfeld YJ, Chitkara U, Hudgins L, Quake SR. Noninvasive diagnosis of fetal aneuploidy by shotgun sequencing DNA from maternal blood. Proc Natl Acad Sci U S A 2008;105:16266-16271.

12. Benn PA, Chapman AR. Practical and ethical considerations of noninvasive prenatal diagnosis. JAMA 2009;301:2154-2156.

13. Report on Prenatal Diagnostic Testing in Victoria 2007. Available at: http://www.health.vic.gov.au/_data/assets/pdf_file/0016/314206/report_ diagnostictest2007.pdf. Accessed October 30, 2009.

14. Report on Prenatal Diagnostic Testing in Victoria 2006. Available at: http://www.health.vic.gov.au/_data/assets/pdf_file/0015/314205/report diagnostictest2006.pdf. Accessed October 30, 2009.

15. Hahn S, Jackson LG, Kolla V, Mahyuddin AP, Choolani M. Noninvasive prenatal diagnosis of fetal aneuploidies and Mendelian disorders: new innovative strategies. Expert Rev Mol Diagn 2009;9:613-621.
16. Lo TK. Screening options for Down syndrome: how women choose in real clinical setting. Prenat Diagn 2009;29:852-856.

17. Cell-free fetal nucleic acids for non-invasive prenatal diagnosis, Report of the UK expert working group, PHG Foundation. Available at: http://www. phgfoundation.org/download/ffdan/ffDNA_report.pdf. Accessed October 28, 2009.

18. Jaques AM, Halliday JL, Francis I, et al. Follow up and evaluation of the Victorian first-trimester combined screening programme for Down syndrome and trisomy 18. BJOG 2007;114:812-818.

19. Jaques AM, Collins VR, Haynes K, et al. Using record linkage and manual follow-up to evaluate the Victorian maternal serum screening quadruple test for Down's syndrome, trisomy 18 and neural tube defects. J Med Screen 2006; $13: 8-13$

20. Caughey AB, Washington AE, Kuppermann M. Perceived risk of prenatal diagnostic procedure-related miscarriage and Down syndrome among pregnant women. Am J Obstet Gynecol 2008;198:333 e331-e338.

21. Tabor A, Vestergaard $\mathrm{CH}$, Lidegaard $\mathrm{O}$. Fetal loss rate after chorionic villus sampling and amniocentesis: an 11-year national registry study. Ultrasound Obstet Gynecol 2009;34:19-24.

22. Ratcliffe S. Long-term outcome in children of sex chromosome abnormalities. Arch Dis Child 1999;80:192-195.

23. Baena N, De Vigan C, Cariati E, et al.; EUROSCAN Working Group. Prenatal detection of rare chromosomal autosomal abnormalities in Europe. Am J Med Genet A 2003;118A:319-327.

24. Franssen MT, Korevaar JC, van der Veen F, Leschot NJ, Bossuyt PM, Goddijn M. Reproductive outcome after chromosome analysis in couples with two or more miscarriages: index [corrected]-control study. BMJ 2006; 332:759-763.

25. Holmgren C, Lacoursiere DY. The use of prenatal ultrasound for the detection of fetal aneuploidy. Clin Obstet Gynecol 2008;51:48-61.

26. Gaudry P, Lebbar A, Choiset A, et al. Is rapid aneuploidy screening used alone acceptable in prenatal diagnosis? an evaluation of the possible role of ultrasound examination. Fetal Diagn Ther 2009;25:285-290.

27. Boormans EM, Birnie E, Bilardo CM, et al. Karyotyping or rapid aneuploidy detection in prenatal diagnosis? The different views of users and providers of prenatal care. BJOG 2009;116:1396-1399.

28. Chan YM, Sahota DS, Leung TY, Choy KW, Chan OK, Lau TK. Chinese women's preferences for prenatal diagnostic procedure and their willingness to trade between procedures. Prenat Diagn 2009;29:870-874.

29. Grimshaw G, Szczepura A, Hulten M, et al. Evaluation of molecular tests for prenatal diagnosis of chromosome abnormalities. Health Technol Assess 2003; $7: 1-146$

30. Ryan M, Diack J, Watson V, Smith N. Rapid prenatal diagnostic testing for Down syndrome only or longer wait for full karyotype: the views of pregnant women. Prenat Diagn 2005;25:1206-1211

31. Kooij L, Tymstra T, Berg P. The attitude of women toward current and future possibilities of diagnostic testing in maternal blood using fetal DNA. Prenat Diagn 2009;29:164-168.

32. van Zwieten MC, Willems DL, Litjens LL, Schuring-Blom HG, Leschot N. How unexpected are unexpected findings in prenatal cytogenetic diagnosis? A literature review. Eur J Obstet Gynecol Reprod Biol 2005;120:15-21.

33. Skotko BG. With new prenatal testing, will babies with Down syndrome slowly disappear? Arch Dis Child 2009;94:823-826. 